\title{
Inferring Multiple Activities of Mobile Users with Activity Algebra
}

\author{
Saguna $^{1,2}$ \\ ${ }^{1}$ Monash University, Melbourne, Victoria, Australia \\ ${ }^{2}$ Luleå University of Technology, SE-971 87, Luleå, Sweden, \\ saguna.saguna@monash.edu
}

\begin{abstract}
This paper proposes a novel multiple activity recognition and reasoning approach where we use on-body sensor information along with other context information to infer mobile user activities which are both concurrent and interleaved. We develop and validate an activity algebra and a complex activity recognition algorithm for detecting these multiple activities. Activities are mapped onto situations using spatio-temporal analysis. We validate our approach by implementing a prototype and performing experiments in different scenarios using mobile devices.
\end{abstract}

Keywords- activity recognition; context-awareness; mobile device; prototype

\section{INTRODUCTION}

The advancement of sensing technology has evoked an increased interest in the field of human activity recognition, for example, in fields like health monitoring, aged care, sports and military [1]. There is progression of this technology to be used in recognising activities of daily living (ADL) which can be personal or professional activities performed at home, work or outdoors. ADL recognition can help in developing smarter environments. Human users multitask and perform highly complex activities as operations which are routinized. A large body of work [2-7] exists in the field of activity analysis which focuses on atomic activities (like sitting, picking a glass). All human activity recognition problems somewhat follow a common approach [1]. This includes the use of sensors, multi-sensor fusion (data-level, feature-level or decision-level), the segmentation of data with relation to time and space, feature extraction and selection, classification of activities, and the use of activity models to infer activities which can be based on a number of techniques such as Bayesian networks [2], hidden Markov models [3], artificial neural networks [4], string-matching algorithms [5], decision trees [6] and the use of semantics [7]. There has been emphasis on order and sequence in building activity models based on machine learning techniques. Recent work using data mining has also approached activity analysis and it differs from machine learning algorithms as it focuses on capturing the varied order of activities $[8,9]$. Activity recognition problem when focussed in special environments like industry and sports are required to have a strong focus on order and sequence of activities. Though sequence is important in activity inference, we identify the problem that ADLs are usually complex activities where the sequence of the atomic activities within these complex activities frequently changes. This change of sequence is inherent in both a single person performing the same activity a second time and different persons performing the same activity. This makes the use of existing activity models tedious to train and sensitive to additions of new activities. They also face problem areas such as activity labelling for large datasets. A new activity model is created each time an activity is added. Thus, there is a need to infer users' complex activities which are concurrent, interleaved and may or may not have a fixed sequence.

\section{RESEARCH CHALLENGES AND CONTRIBUTIONS}

Recognition of complex ADLs is an important research challenge because (1) there is large number of activities to be recognized and (2) the activity sequence varies each time an activity is performed. It is difficult to create activity models for such large number of activities which are highly complex and then to retrain these models for changes in sequence as well as addition of new activities. Activity inference within smart home environments has seen immense interest from research community but there still remains the challenge of inferring complex activities where such environments are not available. The challenge remains to create generic complex activity inference techniques which help human users from all walks of life to take advantage of it with minimal or no training as well as limited domain knowledge.

In this paper, we propose and develop an activity algebra which is used in defining atomic and complex activities. We further develop an algorithm which is used to infer complex concurrent and interleaved activities where context reasoning is performed to infer situations. In doing so, we develop a novel situation- and context-aware activity recognition system $\left(\mathrm{SACAAR}^{1}\right)$. To this extent, we look at activities from the perspective of events, atomic or composite. We then infer situations and link activities to situations. These situations are an extension of the concept of composite events [10]. We develop an event based activity algebra on top of existing research [10] to define activities. Thus, we propose a novel approach for complex activity recognition which can be deployed to infer concurrent as well as interleaved activities. Our key contributions can be summarized as: (1) we develop activity algebra which is used to define atomic and complex activities, (2) we build a complex activity inference algorithm which is used to infer both concurrent and interleaved activities and (3) we build a 
test-bed and prototype to evaluate and validate SACAAR using experimental analysis.

\section{RELATED WORK}

In $[6,11-13]$, activity analysis research focuses on atomic activity inference where the context and sensor information is limited to one or two types of sensory data. We propose an activity inference mechanism that incorporates a comprehensive set of sensor and context information such as accelerometer for physical activity, RFID for object interaction based activities, device activities for laptops and mobile phones, browser activity for websites visited, user location, environmental temperature along with direction of travel and speed of user. The use of multiple sensors can significantly improve accuracy. It can also be used to infer not just atomic but complex activities [6] as well. Hence, we take this approach towards activity inference. We take the work on events presented in [10] as inspiration to define activities and the context spaces theory [14] to infer situations. We build on top of AMIT situation definition language [10] and define atomic and complex activities keeping with the analogy of events. Further, we use the activity algebra defined in section IV and context spaces theory [14] to model activities and map them to situations.

In [15] and [16], surrounding context information and atomic activities are recognized respectively and later shared on a web portal. Our approach differs from the existing work [16] as it is used to solve the challenging problem of inferring complex activities which are concurrent and interleaved. In comparison to $[8,9]$, our approach uses minimal domain knowledge and requires no training of activity models to infer complex activities. Thus, we eliminate the step of collecting large datasets and annotation of this data. Also, for every change in sequence as well as change in the steps of interleaving and concurrency while performing complex activities does not require additional training data in our approach. We infer complex activities from atomic activities and additional context information. Data mining techniques applied to concurrent and interleaved activities in [9] are interesting as they help in searching emerging patterns using frequent itemset mining and find abnormally growing patterns but we differ from this work fundamentally as we use our activity algebra and additional context information as well as a reasoning approach to infer activities. The technique in [9] is used to infer concurrent and interleaved activities but authors highlight that specific characteristics relating to some subjects were not captured when performing complex activities which were interleaved and concurrent. Also, the sliding window approach used at complex activity level in [9] can adversely affect activity recognition as an error in detection of one boundary can lead to further error accumulation during activity recognition.

In [8], activities are discovered using a mining method called discontinuous varied-order mining and then clustering is performed to identify activities. For the inference of activities voted HMM is used and the relative frequencies from sample data are used for training. Though, the techniques applied our interesting, this would still require observing individuals for weeks to create clusters of activities and then human input to identify the clusters relating to different activities. Clustering techniques used in [8] can adversely affect recognition of activities which are performed with significant variation in sequence or if some steps are missed. Authors have highlighted that accuracy can be improved by using context information apart from mere touch sensors in a smart home environment. We highlight that an activity algebra which includes definitions of atomic and complex activities along with reasoning of context and atomic activities, can lead to more accurate and dependable recognition of complex activities. This can be used to identifying key atomic activities within a complex activity. Our approach differs from existing research $[8,9]$ as we use mobile devices and inference of activities is not limited to smart home environments.

\section{ACTIVITY ALGEBRA, SITUATION SPACES AND REASONING OF CONTEXT AND ATOMIC ACTIVITY}

In this section, we propose and investigate the activity algebra for defining and inferring multiple (concurrent and interleaved) complex activities. This is followed by relating situations with activities. We describe how inferring situations can help in inferring activities. We further develop a context and atomic activity reasoning approach to infer complex activities.

DEFINITION1: Atomic activity, $A$ is defined as activity which can be observed from a set of sensors, $\Sigma_{\mathrm{S}}=\left\{\mathrm{s}_{1}, \ldots, \mathrm{s}_{\mathrm{n}}\right\}$, where $n \geq 1$ and the level of granularity can vary based on sensor deployment scenarios. For example,

Case 1: $A_{1}=$ "user is walking" inferred by sensor $s_{1}$ which is an accelerometer placed on the user's waist. Case 2: $A_{1}=$ "user is walking" inferred by sensors $s_{1}, s_{2}, s_{3}$ and $s_{4}$, where $\mathrm{s}_{1}, \mathrm{~s}_{2}, \mathrm{~s}_{3}$ are three accelerometers placed on different parts of the body and $s_{4}$ is a GPS sensor placed on the user which provides individual body part movements and velocity information respectively.

DEFINITION 2: Complex activity, $\mathrm{CA}$ is a tuple $\mathrm{CA}=(\gamma \mathrm{A}$, $\left.\rho C, A_{S} \vee C_{S}, A_{E} \vee C_{E}, T_{S}, T_{E}, T_{L}\right)$ where $\gamma A$ is the sub-set of atomic activities from the complete set of atomic activities, $\Sigma_{A}=\left\{A_{1}, \ldots, A_{n}\right\}, n \geq 1$ and $\rho C$ is the sub-set of context information from the complete set of context information, $\Sigma_{\mathrm{C}}=\left\{\mathrm{C}_{1}, \ldots, \mathrm{C}_{\mathrm{n}}\right\}, \mathrm{n} \geq 1$ which must occur for a complex activity $\mathrm{CA}$ to occur (where the order within $\gamma \mathrm{A}$ is not important), $\left(\mathrm{A}_{\mathrm{S}}, \mathrm{A}_{\mathrm{E}}\right) \in \Sigma_{\mathrm{A}}$ and are the start and end atomic activities respectively, $\left(\mathrm{C}_{\mathrm{S}}, \mathrm{C}_{\mathrm{E}}\right) \in \Sigma_{\mathrm{C}}$ and are the start and end context information respectively. CA can have multiple start and end atomic activities as well as context information, $\mathrm{T}_{\mathrm{S}}$ and $\mathrm{T}_{\mathrm{E}}$ denote the start time and end time of a complex activity. $T_{L}=\left|T_{E}-T_{S}\right|$ is the complex activity lifespan and $\mathrm{T}_{\mathrm{Lmin}}<\mathrm{T}_{\mathrm{L}}<\mathrm{T}_{\mathrm{Lmax}}$ where $\left(\mathrm{T}_{\mathrm{Lmin}}, \mathrm{T}_{\mathrm{Lmax}}\right)$ gives the time range for a complex activity, for example,

Case 1: $\mathrm{CA}_{1}=$ "user is walking to bus stop" is inferred by atomic activity, $\mathrm{A}_{1}=$ user is walking and context 
TABLE I. COMPLEX ACTIVITY EXAMPLE.

\begin{tabular}{|c|c|c|c|c|c|c|c|c|c|}
\hline $\begin{array}{c}C A_{k} \\
\left(\omega_{C A_{k}}^{T}\right)\end{array}$ & $\gamma \mathbf{A}\left(w_{C A_{k}}^{A_{i}}\right)$ & $\rho C\left(w_{C A_{k}}^{C_{i}}\right)$ & $\begin{array}{l}\text { Core } \gamma A \\
\text { and } \rho C\end{array}$ & $\begin{array}{l}\mathbf{A}_{\mathbf{S}} \\
, \mathbf{C}_{\mathbf{S}}\end{array}$ & $A_{E}, C_{E}$ & $\mathbf{T}_{\mathbf{S}}$ & $\mathbf{T}_{\mathbf{E}}$ & $\begin{array}{l}\text { Time } \\
\text { taken } \\
\text { (mins) }\end{array}$ & $\begin{array}{c}\mathrm{T}_{\mathrm{L}} \\
\text { range } \\
\text { (mins) }\end{array}$ \\
\hline $\begin{array}{l}\text { Cooking } \\
\text { omelette for } \\
\text { breakfast in } \\
\text { kitchen } \\
(0.89)\end{array}$ & $\begin{array}{l}\mathrm{A}_{1}: \operatorname{standing}(0.01), \mathrm{A}_{2}: \text { walking }(0.01), \\
\mathrm{A}_{3}: \text { fridge }(0.02), \\
\mathrm{A}_{4}: \operatorname{plate}(0.02), \mathrm{A}_{5}: \operatorname{eggs}(0.16), \\
\mathrm{A}_{6}: \operatorname{frypan}(0.14), \mathrm{A}_{7}: \operatorname{vegetable} \\
\text { drawer(}(0.02), \mathrm{A}_{8}: \operatorname{bowl}(0.12), \\
\mathrm{A}_{9}: \text { whisker }(0.12)\end{array}$ & $\begin{array}{l}\mathrm{C}_{1}: \text { in home } \\
\text { kitchen }(0.12), \\
\mathrm{C}_{2}: \text { morning }(0.12) \text {, } \\
\mathrm{C}_{3}: \text { kitchen light } \\
\text { on }(0.03), \mathrm{C}_{4}: \text { stove } \\
\text { on }(0.12)\end{array}$ & $\begin{array}{l}\text { Core } \gamma \mathrm{A}= \\
\left(\mathrm{A}_{5}, \mathrm{~A}_{6}, \mathrm{~A}_{8},\right. \\
\left.\mathrm{A}_{9}\right) \\
\text { Core } \rho \mathrm{C}= \\
\left(\mathrm{C}_{1}, \mathrm{C}_{2}, \mathrm{C}_{4}\right)\end{array}$ & $\begin{array}{l}\mathrm{C}_{1}, \\
\mathrm{~A}_{5}, \\
\mathrm{~A}_{3}, \\
\mathrm{~A}_{6}, \\
\mathrm{~A}_{10}, \\
\mathrm{~A}_{9}\end{array}$ & $\begin{array}{l}\neg \mathrm{C}_{3}, \\
\mathrm{~A}_{4}, \\
\neg \mathrm{C}_{4}\end{array}$ & 07:06 & $07: 22$ & 16 & $10-20$ \\
\hline
\end{tabular}

information, $\mathrm{C}_{2}=$ user direction towards bus stop from home. Case 2: $\mathrm{CA}_{2}=$ "user is working on presentation at home" is inferred by atomic activities, $A_{2}=$ user is sitting, $A_{3}=$ user is using study desk, $\mathrm{A}_{4}=$ user is detected near laptop, $\mathrm{A}_{5}=$ user is typing in Microsoft Powerpoint application file and context information, $\mathrm{C}_{3}=$ user location is home, $\mathrm{C}_{4}=$ study desk light is on. We further look at an example of concurrent and interleaved activities. Case 3: A user performs the following activities concurrently and interleaved in time. $\mathrm{CA}_{3}=$ "user is reading/writing a document on his/her laptop in room at office", $\mathrm{CA}_{4}=$ "user is browsing the Internet for research articles", $\mathrm{CA}_{5}=$ "user is drinking coffee", $\mathrm{CA}_{6}=$ "user is chatting on IM with friend". We can further perform operations on complex activities such as unions to create complex activities such as $\left(\mathrm{CA}_{3} \cup \mathrm{CA}_{4} \cup \mathrm{CA}_{5}\right)$.

In order to improve accuracy weights can be assigned to atomic activities and context information belonging to each complex activity. The core set of atomic activities and context information which are extremely essential for the occurrence of a complex activity are assigned higher weights while those atomic activities which can be given a miss when successfully performing a complex activity can be assigned lower weights as seen in: table I. We do not consider order of atomic activities while performing complex activities though if required it can be easily accommodated. Also, of interest here is the concept of situations. Humans in a day are part of a number of situations. All activities that are performed by them are part of some situation. By identifying situations we can limit the number of occurring activities to be identified by their linkage to a particular situation. In a situation hierarchy, where situations and sub-situations exist, it is possible to identify situations with a limited set of sensor and context information while complex activity inference would require larger amounts of sensory as well as context data to be processed. We use the context spaces theory [14] to infer situations and then use this knowledge to further infer activities. Also, a complex activity can occur in time such that it traverses through more than one situation during its completion. The situations can be inferred from spatio-temporal information as well as other context information. To infer complex activities, we propose reasoning of context and atomic activity information. Each complex activity has a list of atomic activities, $\gamma \mathrm{A}$ and a list of context information, $\rho \mathrm{C}$ as mentioned in Definition 2. Each atomic activity, $A_{i}$ and context information, $C_{i}$ is assigned a particular weight, $w_{C A_{k}}^{A_{i}}$ and $w_{C A_{k}}^{C_{i}}$ respectively, corresponding to its importance in relation to the occurrence of a complex activity $\mathrm{CA}_{\mathrm{k}}$. The sum of all the weights, $\omega_{C A_{k}}$ for each $\mathrm{CA}_{k}$ is 1 . If $\mathrm{A}_{\mathrm{i}}$ or $\mathrm{C}_{\mathrm{i}}$ do not occur for a particular $\mathrm{CA}_{k}$ then $w_{C A_{k}}^{A_{i}}=0$ and $w_{C A_{k}}^{C_{i}}=0$. The sum of all the weights, $\omega_{C A_{k}}$ for all occurring atomic activities and context information needs to be above a threshold, $\omega_{C A_{k}}^{T}$ in order for $\mathrm{CA}_{k}$ to occur successfully. If the sum of weights, $\omega_{C A_{k}}$ is less than the threshold, $\omega_{C A_{k}}^{T}$ then 1) it implies that the activity was started but abandoned in between, 2) it implies that the core set of atomic activities and context information for that particular $\mathrm{CA}_{k}$ did not occur. Thus,

$\omega_{C A_{k}}=\sum_{i=1}^{N} w_{C A_{k}}^{A_{i}}+\sum_{i=1}^{n} w_{C A_{k}}^{C_{i}}-E q(1)$ where $0 \leq \omega \leq 1$, $\sum_{i=1}^{N} w_{C A_{k}}^{A_{i}}=\left(w_{C A_{k}}^{A_{1}}+\cdots+w_{C A_{k}}^{A_{N}}\right)-E q(2)$ and

$\sum_{i=1}^{N} w_{C A_{k}}^{C_{i}}=\left(w_{C A_{k}}^{C_{1}}+\cdots+w_{C A_{k}}^{C_{N}}\right)-E q(3)$ and

$w_{C A_{k}}^{A_{i}}=0$ and $w_{C A_{k}}^{C_{i}}=0$, if $\mathrm{A}_{\mathrm{i}}$ and $\mathrm{C}_{\mathrm{i}}$ do not occur for $\mathrm{CA}_{k}$ and $\omega_{C A_{k}} \geq \omega_{C A_{k}}^{T}-E q(4)$ for any $\mathrm{CA}_{k}$ to have occurred successfully. We further demonstrate the use of weights and our activity algebra in a single example due to lack of space. Working Example: $\mathrm{CA}_{1}=$ Cooking omelette for breakfast in kitchen is a complex activity as shown in table I. We use our activity algebra along with our reasoning approach to define and infer $\mathrm{CA}_{l}$. We define the $\mathrm{CA}_{l}=\left(\gamma \mathrm{A}, \rho \mathrm{C}, \mathrm{A}_{\mathrm{S}} \vee \mathrm{C}_{\mathrm{S}}, \mathrm{A}_{\mathrm{E}} \vee \mathrm{C}_{\mathrm{E}}\right.$, $\mathrm{T}_{\mathrm{S}}, \mathrm{T}_{\mathrm{E}}, \mathrm{T}_{\mathrm{L}}$ ) tuple based on domain knowledge in a way that it is straightforward for users to define the tuple themselves by using the type of atomic activities and context information available. The assignment of weights is based on the importance of each $\mathrm{A}_{\mathrm{i}}$ and $\mathrm{C}_{\mathrm{i}}$ for a corresponding $\mathrm{CA}_{l}$, for example, here the highest weight is assigned to $\mathrm{A}_{5}=0.16$ followed by $\mathrm{A}_{6}=0.14$ which are very important atomic activities for cooking an omelette. Also, $\mathrm{C}_{1}, \mathrm{C}_{2}$ and $\mathrm{C}_{4}$ are assigned weights of 0.12 as they are all of equal importance in the process of performing $\mathrm{CA}_{1}$ while $\mathrm{C}_{3}$ may or may not be switched on depending on time of day. In this case, $\omega_{C A_{k}}$ should be greater than the threshold $\omega_{C A_{k}}^{T}=0.89$ for $\mathrm{CA}_{l}$ to have successfully occurred. It is important to note that we take temporal context into consideration as part of the complete set of context information, $\Sigma_{\mathrm{C}}$ and the non occurrence of an atomic activity is represented as $\neg \mathrm{A}$. 


\section{ACTIVITY RECOGNITION USING SACAAR SYSTEM}

Our proposed SACAAR system is used for complex activity recognition. The sensory layer collects sensor and context information from different wearable sensors, virtual sensors and other sensors in the environment. In the activity inference layer, for atomic activity inference we use decision trees to infer body motion, temporal analysis to infer RFID object interaction, device activity and browser activity. Situation-aware activity reasoning is done to infer situations by the spatio-temporal context complexity filter (CCF). Table II shows the pseudo code for the complex activity recognition algorithm which consists of finding the start atomic activity and then setting a time window of the size of the lifespan $T_{L}$ for each matched $A_{S} \vee C_{S}$ belonging to a $C_{k}$. We then look for matching $\gamma \mathrm{A}, \rho \mathrm{C}$ and $\mathrm{A}_{\mathrm{E}} \vee \mathrm{C}_{\mathrm{E}}$ within the time window for each $\mathrm{CA}_{\mathrm{k}}$. We compute the $\omega_{C A_{k}}$ using equation (1) in section IV and then check against $\omega_{C A_{k}}^{T}$ as shown in equation (4). If the condition is matched $\mathrm{CA}_{k}$ is inferred successfully. All time windows run in parallel and all incoming $\mathrm{A}_{i}$ and $\mathrm{C}_{i}$ matching each $\mathrm{CA}_{k}$ are added to them till a successful match is found. The weights are added at runtime after each addition.

TABLE II. COMPLEX ACTIVITY RECOGNITION ALGORITHM.

INPUT: $\mathrm{A}_{\mathrm{i}}, \mathrm{C}_{\mathrm{i}}, \mathrm{S}_{\mathrm{i}}$; OUTPUT: $\mathrm{CA}_{\mathrm{k}}$ Pseudocode:

findStartAtomicActivity $\left(\mathrm{A}_{\mathrm{i}}, \mathrm{C}_{\mathrm{i}}\right)$

$1 .\left\{\right.$ check for current $S_{i}$

2. findComplexActivitiesList $\left(\mathrm{S}_{\mathrm{i}}\right)$

3. foreach $\left(\mathrm{CA}_{\mathrm{k}}\right)\{$

4. if $\left(\mathrm{A}_{\mathrm{i}}==\mathrm{A}_{\mathrm{S}}\right)$

5. $\quad \operatorname{add}\left(\mathrm{CA}_{\text {list }} \leftarrow \mathrm{CA}_{\mathrm{i}}=\left(\gamma \mathrm{A}, \rho \mathrm{C}, \mathrm{A}_{\mathrm{S}}, \mathrm{A}_{\mathrm{E}}, \mathrm{C}_{\mathrm{S}}, \mathrm{C}_{\mathrm{E}}, \mathrm{T}_{\mathrm{L}}\right)\right)$ \}//foreach

\section{6. return $\left.\mathrm{CA}_{\text {list }}\right\} / /$ findStartAtomicActivity}

findComplexActivity $\left(\mathrm{A}_{\mathrm{i}}, \mathrm{C}_{\mathrm{i}}\right)$

1. $\left\{\right.$ foreach $\left(\mathrm{CA}_{\text {list }} \leftarrow \mathrm{CA}_{\mathrm{k}}\right)$ \{

2. while (time counter $\left.<\mathrm{T}_{\mathrm{Lmax}} \mathrm{i}\right)\{$

3. if $\left(\mathrm{A}_{\mathrm{i}}==\right.$ element in $\left.\gamma \mathrm{A}_{\mathrm{i}}\right)$

4. $\quad$ add $\mathrm{A}_{\mathrm{i}} \rightarrow \gamma \mathrm{A}_{\mathrm{i}}$ and recalculate $w_{C A_{k}}^{A_{i}}$

5. if $\left(\mathrm{C}_{\mathrm{i}}==\right.$ element in $\left.\rho \mathrm{C}_{\mathrm{i}}\right)$

6. $\quad$ add $\mathrm{C}_{\mathrm{i}} \rightarrow \rho \mathrm{C}_{\mathrm{i}}$ and recalculate $w_{C A_{k}}^{C_{i}}$

7. if $\left(\mathrm{A}_{\mathrm{E}}, \mathrm{C}_{\mathrm{E}}\right.$ found for $\left.\mathrm{CA}_{\mathrm{i}}\right) \&\left(\rho \mathrm{C}_{\mathrm{i}}\right.$ and $\gamma \mathrm{A}_{\mathrm{i}}$ are complete

and $\left.\left.\omega_{C A_{k}} \geq \omega_{C A_{k}}^{T}\right)\right)$

8. found $\left.\mathrm{CA}_{\mathrm{k}}\right\} / /$ while $\} / /$ foreach

9. return $\mathrm{CA}_{\mathrm{k}}$. $\} / /$ findComplexActivity

\section{RESULTS}

We performed experiments on two subjects for three weeks with an average of seven hours daily. The experiments were performed usually in the time range of 8:00 am to $12: 00 \mathrm{pm}$ and from $1: 30 \mathrm{pm}$ to $5: 30 \mathrm{pm}$. We identified 16 complex activities and used our activity algebra to define them. We have built a test-bed comprising of different sensing technology as mentioned previously in section V. Our algorithm performed with an overall accuracy of $88.5 \%$. We performed online inferencing using our test-bed. The users were given complete freedom to perform the previously defined complex activities in any interweaved and concurrent manner and in any order. It is important to note that our approach does not require training data to create activity models. It only uses domain knowledge to define complex activities and create links to atomic activities.

\section{CONCLUSION AND FUTURE WORK}

In this paper, we propose, develop and evaluate SACAAR system along with our activity algebra to infer concurrent and interleaved complex activities. We perform activity and context reasoning to infer complex activities. In future, we intend to extend on our activity algebra, reasoning approach as well. The SACAAR system can also be extended to infer complex activities which involve multiple users.

\section{REFERENCES}

[1] G.-Z. Yang, B. Lo, and S. Thiemjarus, Body Sensor Networks: Springer London, 2006.

[2] F. Albinali, N. Davies, and A. Friday, "Structural Learning of Activities from Sparse Datasets," in Pervasive Computing and Communications, Fifth Annual IEEE International Conference on, 2007, pp. 221-228.

[3] M. Philipose, K. P. Fishkin, M. Perkowitz, D. J. Patterson, D. Fox, H. Kautz, and D. Hahnel, "Inferring activities from interactions with objects," Pervasive Computing, IEEE, vol. 3, pp. 50-57, 2004.

[4] K. Van Laerhoven, "Combining the Self-Organizing Map and K-Means Clustering for On-Line Classification of Sensor Data," in Artificial Neural Networks - ICANN 2001, 2001, pp. 464-469.

[5] T. Stiefmeier, D. Roggen, and G. Troster, "Gestures are strings: efficient online gesture spotting and classification using string matching," in Proceedings of the ICST 2nd international conference on Body area networks Florence, Italy, 2007.

[6] L. Bao, "Physical Activity Recognition from Acceleration Data under Semi-Naturalistic Conditions," in Department of Electrical Engineering and Computer Science: Massachusetts Institute of Technology, 2003.

[7] D. Riboni and C. Bettini, "Context-Aware Activity Recognition through a Combination of Ontological and Statistical Reasoning," in Ubiquitous Intelligence and Computing, 2009, pp. 39-53.

[8] P. Rashidi, D. Cook, L. Holder, and M. Schmitter-Edgecombe, "Discovering Activities to Recognize and Track in a Smart Environment," Knowledge and Data Engineering, IEEE Transactions on, vol. 23, no. 4, pp. 527-539, April 2011.

[9] G. Tao, W. Zhanqing, T. Xianping, P. Hung Keng, and L. Jian, "epSICAR: An Emerging Patterns based approach to sequential, interleaved and Concurrent Activity Recognition," in Pervasive Computing and Communications, IEEE International Conference on, 2009, pp. 1-9.

[10] A. Adi and O. Etzion, "Amit - the situation manager," The VLDB Journal, vol. 13, pp. 177-203, 2004.

[11] M. Stikic, T. Huynh, K. Van Laerhoven, and B. Schiele, "ADL recognition based on the combination of RFID and accelerometer sensing," in Pervasive Computing Technologies for Healthcare, 2008. PervasiveHealth 2008. Second International Conference on, 2008, pp. 258-263.

[12] S. Thomas, L. Clemens, R. Daniel, J. Holger, O. Georg, and T. Gerhard, "Event-Based Activity Tracking in Work Environments," in Proceedings of the 3 rd IFAWC, 2006, pp. 91-102.

[13] O. Amft and G. Troster, "Recognition of dietary activity events using onbody sensors," Artif. Intell. Med., vol. 42, pp. 121-136, 2008.

[14] A. Padovitz, S. W. Loke, and A. Zaslavsky, "Towards a Theory of Context Spaces," Proceedings of the Second IEEE Annual Conference on Pervasive Computing and Communications Workshops, 2004.

[15] Nokia, "Sensing the World with Mobile Devices," White Paper, Nokia Technology Insights series December 2008.

[16] T. Choudhury, S. Consolvo, B. Harrison, J. Hightower, A. LaMarca, L. LeGrand, A. Rahimi, A. Rea, G. Bordello, B. Hemingway, P. Klasnja, K. Koscher, J. A. Landay, J. Lester, D. Wyatt, and D. Haehnel, "The Mobile Sensing Platform: An Embedded Activity Recognition System," Pervasive Computing, IEEE, vol. 7, pp. 32-41, 2008. 